\title{
Perdão Conjugal: Uma Explicação a partir dos Valores Humanos
}

\author{
Patricia Nunes da Fonsêca \\ Centro de Educação, Departamento de Psicopedagogia, Universidade Federal da Paraíba, \\ João Pessoa, PB, Brasil \\ Bruna de Jesus Lopes ${ }^{1}$ \\ Centro de Ciências Humanas, Letras e Artes, Universidade Federal da Paraíba, \\ João Pessoa, PB, Brasil \\ Estefânea Élida da Silva Gusmão \\ Núcleo de Avaliação Psicológica em Saúde, Departamento de Psicologia, Universidade \\ Federal do Ceará, Fortaleza, CE, Brasil \\ Viviany Silva Araújo Pessoa \\ Centro de Educação, Departamento de Psicopedagogia, Universidade Federal da Paraíba, \\ João Pessoa, PB, Brasil \\ Ricardo Neves Couto \\ Centro de Ciências Humanas, Letras e Artes, Universidade Federal da Paraíba, \\ João Pessoa, PB, Brasil \\ Maria Izabel Fernandes da Silva \\ Centro de Educação, Departamento de Psicopedagogia, Universidade Federal da Paraíba, \\ João Pessoa, PB, Brasil
}

\begin{abstract}
Resumo
Teve-se como objetivo conhecer a relação entre o perdão conjugal e os valores humanos, bem como verificar o poder deste último em predizer a remissão marital. Para isso, contou-se com 313 pessoas, casadas ou em união estável, do estado da Paraíba (70\%) e do Piauí (30\%). As idades variaram de 18 a 70 anos $(M=35,00 ; D P=11,41)$, sendo a maioria do sexo feminino $(67,7 \%)$. Estes responderam os instrumentos: Escala de Perdão Conjugal, Questionário de Valores Básicos e um Questionário Sociodemográfico. Foram executadas duas Análises de Regressão Linear Múltipla (método Stepwise). Na primeira, tendo como variáveis previsoras as subfunções valorativas, constatou-se que a subfunção interativa explica o perdão conjugal. Contribuindo de forma direta na explicação da concessão do perdão entre casais. Na segunda, fixaram-se como variáveis explicativas os tipos de orientação. Esta revelou que os valores sociais explicam a remissão matrimonial, contribuindo de forma direta. Diante destes resultados, conclui-se que os indivíduos que priorizam valores sociais encontram-se mais predispostos a conceder o perdão dentro do casamento.
\end{abstract}

Palavras-chave: Perdão, valores humanos, conjugalidade.

1 Endereço para correspondência: Universidade Federal da Paraíba, Bases Normativas do Comportamento Social, Centro de Ciências Humanas, Letras e Artes, João Pessoa, PB, Brasil 58059-900. Fone: (+55) (83) 32167444. E-mail: pnfonseca.ufpb@gmail.com, bruna_lopespsi@hotmail.com, estefanea@gmail.com, viviany. pessoa@gmail.com, r.nevescouto@gmail.com e isabelfernandes_silva@hotmail.com 


\title{
Marital Forgiveness: An Explanation Based on Human Values
}

\begin{abstract}
This study aimed to understand the relationship between marital forgiveness and human values and to discover the latter's power to predict whether a marriage will recover. The study included 313 participants who were married or in a long-term relationship, 70\% from the state of Paraíba and 30\% from the state of Piauí. The participants' ages ranged from 18 to 70 years $(M=35.0 ; S D=11.41)$, the majority being female (67.7\%). The participants answered three questionnaires: the Marital OffenceSpecific Forgiveness Scale, the Basic Values Questionnaire, and a sociodemographic questionnaire. Two stepwise multiple linear regression analyses were conducted. The first, which took the value subfunctions as the predictive variables, found that the interactive subfunction explains marital forgiveness, directly contributing to the explanation of whether forgiveness is granted between couples. The second analysis took the type of orientation as explanatory variables. It found that social values directly contribute to explaining whether the marriage recovers. In light of these results, one may conclude that individuals who prioritize social values are more disposed to grant forgiveness within a marriage.
\end{abstract}

Keywords: Forgiveness, human values, marital relationship.

\section{Perdón Civil: Una Explicación a partir de los Valores Humanos}

\section{Resumen}

Se objetivó conocer la relación entre perdón conyugal y valores humanos, y se buscó verificar el poder de este último para predecir la remisión marital. Se contó con 313 personas, casadas o en unión estable, del estado de Paraíba (70\%) y Piauí (30\%). Las edades oscilaron entre 18 y 70 años $(M=35,00 ; D E=$ 11,41), la mayoría mujeres (67,7\%). Ellos respondieron: Escala de Perdón Conyugal, Cuestionario sobre los Valores Básicos y el Cuestionario sociodemografico. Se realizaron dos Análisis de Regresión Lineal Múltiple (método Stepwise). En el primero, se considero como las variables predictoras las subfunciones valorativas, se encontró que la subfunción interactiva explica el perdón conyugal. Contribuyendo directamente en la explicación de la concesión del perdón entre las parejas. En el segundo, se fijaron como variables explicativas los tipos de orientación. Esto reveló que los valores sociales son responsables por explicar la remisión marital, lo que contribuye directamente. Teniendo en cuenta estos resultados, se concluye que las personas que dan prioridad a los valores sociales son más propensos a conceder el perdón en el matrimonio.

Palabras clave: Perdón, valores humanos, conyugalidad.

O casamento, segundo Bueno, Souza, Monteiro e Teixeira (2013), pode ser compreendido como a emergência de uma nova geração e início de um novo núcleo doméstico. Nesta relação, os impasses do dia a dia não são passíveis de serem impedidos, todavia a forma com que o casal lida com as adversidades pode contribuir com a instabilidade ou a harmonia do relacionamento (Wagner \& Mosmann, 2012).
Fincham, Hall e Beach (2006) apontam o perdão como uma excelente estratégia para a manutenção de relacionamentos conjugais, o qual contribui para a redução do impacto da percepção negativa do outro, aumento da resolução de conflitos e estabilidade do matrimônio. Contudo, apesar de o perdão ser reconhecido há tempos como um fator de grande importância para a conservação dos relacionamentos, somen- 
te nos últimos anos chamou a atenção de pesquisadores (Lin et al., 2014; Peets, Hodges, \& Salmivalli, 2013; Strelan, McKee, Claic, Cook, \& Shaw, 2013), em decorrência dos benefícios provenientes do ato de perdoar, a saber: bem-estar (Allemand, Steiner, \& Hill, 2013), saúde mental e física (Calo-Blanco, 2014), estabilidade emocional (Lander, 2012) e satisfação conjugal (Witvliet, 2001).

A crescente investigação desta temática tem levado ao desenvolvimento de estudos que buscam conhecer sua relação com outras variáveis a exemplo de pensamento moral (Abreu, 2013), satisfação com a saúde geral (Sales, 2014) e valores humanos (Barbosa, 2015). Este último construto, atualmente, tem conduzido um campo de grande investigação, principalmente porque tem se apresentado como uma variável explicadora de vários fenômenos sociopsicológicos (Bardi \& Schwartz, 2003), a exemplo do poliamor (Freire, 2015) e disposição para perdoar (Barbosa, 2015).

Em estudo recente sobre a disposição para perdoar e os valores humanos foi verificado, por meio de uma medida explicita, correlações negativas entre a disposição para perdoar e os valores pessoais (realização e experimentação), ou seja, quanto mais os indivíduos priorizarem valores como prazer, êxito e poder, menos disposição terão para perdoar. Não obstante, quando foi empregada medidas implícitas, os resultados apontaram uma correlação direta entre a disposição para perdoar e os valores sociais (normativo e interativo; Barbosa, 2015).

Contudo, apesar do relacionamento entre valores e perdão ter sido avaliado por Barbosa (2015), cabe destacar que tal estudo foi investigado em amostras extraídas da população geral e acadêmica. Diante dessa generalidade, levantou-se o questionamento de como essas variáveis se comportariam em amostras específicas, a exemplo de casais, especificamente, na concessão do perdão ao parceiro transgressor? Frente a esse panorama, o presente artigo versa sobre o perdão conjugal e a sua relação com os valores humanos.

\section{Perdão Conjugal}

O perdão pode ser definido como a vontade de uma pessoa em deixar de lado o seu direito de ressentimento, julgamento negativo e comportamento indiferente com seu ofensor, nutrindo compaixão, generosidade e amor para com o mesmo (Enright, Rique, \& Coyle, 2000). Portanto, perdoar pode ser entendido como um processo de transformação, cujas respostas negativas e vingativas são substituídas por positivas (McCullough et al., 1998).

Fincham, Paleari e Regalia (2002) compreendem o perdão como uma mudança de motivação que ocorre dentro das relações interpessoais, ou seja, a remissão consiste em um processo em que a motivação à vingança e à evasão são abrandadas, e a motivação benevolente e conciliatória, para com o agressor, são elevadas. Nesse sentido, o perdão pode ser entendido por uma estrutura de duas dimensões, a saber: (a) dimensão negativa, caracterizada por desejos de vingança ou punição ao ofensor; e (b) dimensão positiva, marcada pelo aumento da empatia e libertação da raiva, ocasionando uma maior predisposição à remissão (Fincham \& Beach, 2002; Worthington, 2003). Levando em consideração essas dimensões, Worthington (2005) sugere que a concessão da remissão se dá, nas relações não-contínuas, pela redução da dimensão negativa. Contudo, Fincham et al. (2006) aludem que, nas relações contínuas, faz-se necessário uma diminuição da dimensão negativa e um aumento da dimensão positiva, simultaneamente, para que o perdão seja conferido.

No casamento, o entendimento do perdão não se difere das demais relações interpessoais. A princípio, é necessário que um dos cônjuges se sinta ferido ou injustiçado pelo outro (Enright \& Coyle, 1998), sendo tal ato avaliado como intencional ou, no mínimo, resultado de um descuido (Downie, 1965). Em seguida, a vítima passa a compreender o seu direito de se posicionar de forma negativa para com o ofensor. Este, por sua vez, caso queira a remissão, deve-se mostrar arrependido e paciente, para esperar o abrandamento dos pensamentos, sentimentos e compor- 
tamentos negativos do outro para consigo (North, 1998).

A existência de situações instáveis no matrimônio, provenientes de transgressões (e.g., traições, agressão física e verbal), podem originar emoções negativas resultando, até mesmo, do rompimento das relações conjugais (Fincham, Beach, \& Davila, 2004). Nesse contexto, o perdão pode ser visto como um meio para evitar dissoluções matrimoniais, uma vez que afeta de forma direta e positiva a satisfação conjugal (Chung, 2014; Pelucchi, Paleari, Regalia, \& Fincham, 2013), a resolução de conflito (Fife, Weeks, \& Stellberg-Filbert, 2013), a longevidade (Toussaint, Owen, \& Cheadle, 2012) e a qualidade do casamento (Fincham, Beach, \& Davila, 2007).

Contudo, vale destacar que, apesar de a literatura crescente (Braithwaite, Selby, \& Fincham, 2011; Calo-Blanco, 2014; Hall \& Fincham, 2006; Pelucchi et al., 2013) sobre o perdão conjugal, ainda há a necessidade de se desenvolver novas pesquisas, visando contribuir com o arcabouço científico acerca da temática. $\mathrm{O}$ presente estudo, por exemplo, objetiva verificar o poder dos valores humanos em predizer a disposição dos cônjuges, vítimas de ofensa, a concederem a remissão.

\section{Teoria Funcionalista dos Valores Humanos}

A Teoria Funcionalista dos Valores Humanos, proposta por Gouveia (1998, 2003, 2013), consiste em um modelo parcimonioso e integrador, com foco nas funções dos valores. Segundo o autor (Gouveia, 1998, 2003, 2013), os valores possuem duas funções consensuais, que são: (a) guiar as ações humanas (tipo de orientação; Rokeach, 1973; Schwartz, 1992) e (b) expressar suas necessidades (tipo de motivador; Inglehart, 1977; Maslow, 1954).

As duas dimensões funcionais dos valores compõem dois eixos principais na representação da estrutura proposta: o eixo horizontal, que corresponde ao tipo de orientação, e o eixo vertical, que representa o tipo motivador. Quanto ao tipo de orientação, os valores podem ser: sociais, cujas pessoas guiadas por eles são centradas na sociedade e na convivência com os demais; pessoais, os indivíduos que priorizam esses valores tendem a ser mais egocêntricos e a buscarem alcançar seus próprios interesses e benefícios; e centrais, sendo estes uma inovação do modelo, que figuram entre os valores sociais e pessoais, apresentando congruência com ambos. Desta forma, os valores centrais não se restringem à dicotomia de interesses autocentrados (foco intrapessoal) ou altercentrados (interpessoal); representando seu caráter central ou adjacente em relação aos demais valores, caracterizando-se como uma espécie de "espinha dorsal" (Gouveia, 2013).

Quanto ao tipo de motivador, os valores podem ser classificados em: materialista (pragmático), que implica em uma orientação dirigida a objetivos específicos e regras normativas, são valores característicos de pessoas que têm um pensamento voltado para aspectos biológicos de sobrevivência (e.g., saúde, estabilidade); e idealista (humanitário), que, ao contrário dos valores materialistas, são baseados em princípios e ideias mais abstratas, que se encontram associados à criatividade e à abertura de espírito, sugerindo assim, uma menor dependência de bens materiais (e.g., conhecimento, maturidade; Gouveia, Milfont, \& Guerra, 2014).

O cruzamento desses dois eixos resulta em seis subfunções valorativas: (a) experimentação (pessoal-humanitário), compreende a necessidade fisiológica de satisfação, em sentido amplo, sendo composta pelos valores de prazer, emoção e sexualidade; (b) realização (pessoal-materialista), abarca as necessidades de autoestima, constituída pelos valores êxito, poder e prestígio; (c) existência (central-materialista), refere-se às necessidades fisiológicas mais básicas e à necessidade de segurança, constituída pelos valores de saúde, sobrevivência e estabilidade pessoal; (d) suprapessoal (central-humanitário), alude as necessidades de estética e cognição, formada pelos valores de conhecimento, maturidade e beleza; (e) interativa (social-humanitário), representa as necessidades de pertença, amor e afiliação, composta pelos valores de afetividade, apoio social e convivência; e (f) normativa (social-materialista), reflete a importância de preservar a cultura 
e as normas convencionais, representada pelos valores de religiosidade, tradição e obediência (Gouveia, 2013).

O modelo proposto por Gouveia (1998) vem apresentando uma relação apropriada com diversos construtos, a exemplo, da disposição para perdoar (Barbosa, 2015) além de demonstrar indicadores psicométricos adequados a nível nacional e internacional (e.g., Freire, 2015; Melo, 2014; Monteiro, 2014; Nascimento, 2015), Todavia, não visa substituir outras teorias existentes, mas ser mais uma opção teórica para o desenvolvimento de estudos na área.

Diante do exposto, constata-se que o modelo aqui enfatizado exibe subsídios que motivam a sua utilização na presente pesquisa. Esperando assim, que os valores sociais (Hipótese 1) e centrais (Hipótese 2), bem como suas respectivas subfunções, expliquem diretamente o perdão dentro do relacionamento matrimonial, e que os valores pessoais (Hipótese 3 ) e suas subfunções expliquem o mesmo construto inversamente. Outras hipóteses merecem ser registradas, a saber: haverá diferença entre as médias dos participantes nas duas dimensões do perdão conjugal quanto as seguintes variáveis: (a) sexo, as mulheres pontuariam mais do que os homens (Hipótese 4); (b) idade (Hipótese 5), pessoas mais velhas com maiores magnitudes; (c) nível de religiosidade (Hipótese 6), participantes com maior nível religioso perdoarão mais alto; (d) tempo de união (Hipótese 7), cônjuges com mais tempo de união perdoarão mais; (e) nível de escolaridade (Hipótese 8), que pessoas com maior nível de escolaridade pontuarão mais em perdão.

\section{Método}

\section{Participantes}

Contou-se com uma amostra não probabi- lística, por conveniência, composta por 313 pessoas, casadas ou em união estável, do estado da Paraíba $(n=219 ; 70 \%)$ e do Piauí $(n=94$; $30 \%)$. As idades variaram de 18 a 70 anos $(M=$ $35,00 ; D P=11,41)$, sendo a maioria do sexo feminino $(n=212 ; 67,7 \%)$, de classe média baixa $(n=113 ; 36,1 \%)$, com filhos $(n=229 ; 73,6 \%)$, com pós-graduação $(n=79 ; 25,2 \%)$, e com tempo de relacionamento variando de 1 a 3 anos ( $n$ $=93 ; 29,7 \%)$. Estes se declaravam medianamente religiosos $(M=3,67 ; D P=1,19)$ ao responderem uma escala que variava de 1 (totalmente não religioso) a 5 (totalmente religioso).

\section{Instrumentos}

Os participantes responderam um questionário composto pelos instrumentos a seguir:

Escala de Perdão Conjugal (MOFS): elaborada por Paleari, Regalia e Fincham (2009) e adaptada ao contexto brasileiro por Lopes, Fonsêca, Medeiros, Almeida e Gouveia (2016). Esta escala é composta por 10 itens, divididos, originalmente, da seguinte maneira: quatro para avaliar as motivações benevolentes (e.g., Item 2. Embora ele(a) tenha me machucado, eu definitivamente deixei o que aconteceu de lado, para que pudéssemos continuar nosso relacionamento) e seis para as motivações de ressentimento e vingança (e.g., Item 3. Desde que meu/minha maridolesposa se comportou dessa maneira, eu fico irritado(a) com ele (a) mais facilmente). Todos são respondidos em escala de sete pontos, tipo Likert, variando de 1 (Discordo Totalmente) a 7 (Concordo Totalmente). A versão adaptada ao Brasil apresentou evidências aceitáveis de fidedignidade (Benevolência: alfa de Cronbach $=$ $0,65, r_{\text {m.i }=0,31 \text { e Confiabilidade Composta }=0,66 \text {; Evitação- Ressenti- }}$ mento: alfa de Cronbach $=0,80, r_{\mathrm{m} . \mathrm{i}=0,40 \text { e Confiabilidade }}$ Composta $=0,81$; Lopes et al., 2016).

Questionário de Valores Básicos (QVB18): em sua versão atualizada, sendo este formado por 18 itens, ou valores específicos (Gouveia, 2013). Para cada item são apresentados dois descritores, procurando representar o conteúdo inerente do valor (e.g., saúde é descrito no Item 9 como "preocupar-se com sua saúde antes de ficar doente" e "não estar enfermo"). Estes são respondidos em uma escala de sete pontos variando de 1 (Totalmente não Importante) a 7 (Totalmente Importante). O instrumento apresentou no estudo de Medeiros (2011) em todas as regiões do Brasil alfas variando de 0,48 (interativa) a 0,63 (normativa), e o índice de homogeneidade (r.m.i) com amplitude de 0,24 (interativa) a 0,38 (normativa) para o contexto brasileiro; 
além de apresentar bons indicadores de ajustes $\left[\chi^{2}=949,75\right.$, GFI $=0,92, \mathrm{CFI}=0,81 ; \mathrm{RMSEA}=$ $0,07(90 \% \mathrm{IC}=0,07-0,08)]$.

Questionário Sociodemográfico: os participantes responderam a uma lista de perguntas de cunho demográfico, a saber: (a) idade; (b) sexo; (c) o tempo de união, sendo esta classificada em uma escala intervalar: 1- 3 anos; 4 - 7 anos; 8 13 anos; 14 - 16 anos; e acima de 17 anos; (d) quantidade de filhos; (e) escolaridade - Ensino Fundamental Completo, Ensino Fundamental Incompleto, Ensino Médio Completo, Ensino Médio Incompleto, Ensino Superior Completo, Ensino Superior Incompleto e Pós Graduação; (f) percepção quanto ao nível de religiosidade, expresso em um único item, respondido em uma escala de 5 pontos, variando de 1 (totalmente não religioso) a 5 (totalmente religioso); (g) classe social; e (h) renda familiar, respondido em uma escalar de 6 pontos [(1) De 0 a R \$788,00; (2) De R\$ 789,00 a R\$ 1.576,00; (3) De R \$ 1.577,00 a $\mathrm{R} \$ 2.364,00$; (4) De R\$2.365,00 a R \$ 4.728,00; (5) De R \$ 4.729,00 a R \$ 7.092,00; (2) Acima de $\mathrm{R} \$ 7.093,00]$.

\section{Procedimentos de Coleta de Dados}

A coleta de dados se deu de duas maneiras: a lápis e papel e on-line. Há evidências de uma adequada equivalência entre dados coletados presencialmente, com o tradicional método lápis e papel, e coletados on-line (Brock, Barry, Lawrence, Dey, \& Rolffs, 2012). Na primeira, a amostra $(n=143)$ foi coletada em locais públicos, a exemplo de praças, parques e shoppings. Na segunda, contou-se com um questionário aplicado através da plataforma online Google Drive. Nesta coleta, fez-se uso das redes sociais (e.g., facebook) para ter acesso aos potenciais participantes $(n=170)$. Com as coletas obteve-se um total de 313 participantes. Em ambas aplicações, tomou-se o cuidado de informar o objetivo geral do estudo e clarificar o caráter voluntário da pesquisa, além de informar que a participação não traria nenhum tipo de benfeitoria ou prejuízo, deixando claro o poder do participante de desistir a qualquer momento sem nenhuma perda. O tempo mé- dio de resposta foi de aproximadamente 15 minutos.

\section{Análise de Dados}

Para tabulação e análise dos dados, foi utilizado o pacote estatístico SPSS, versão 20, que foi empregado para realizar estatísticas descritivas (e.g., mediana, média e desvio padrão), Análises de Variância Multivariadas (MANOVAs), Correlações ( $r$ de Pearson) e Regressões Lineares Múltiplas (Método Stepwise).

\section{Procedimentos Éticos}

A princípio, o projeto foi elaborado e submetido ao Comitê de Ética em Pesquisa do Centro de Ciências da Saúde da Universidade Federal da Paraíba (CEP/CCS), tendo sido aprovado sob o Protocolo 0150/15. Por fim, destaca-se que a pesquisa foi realizada seguindo todas as orientações, decretadas na Resolução 466/12 do Conselho Nacional de Saúde, sobre pesquisas com seres humanos.

\section{Resultados}

\section{Correlatos entre Perdão Conjugal e Valores Humanos}

Visando conhecer como as dimensões da Escala de Perdão Conjugal (MOFS) e o fator geral (computado por meio da somatória dos dois itens das dimensões benevolente e evitação - ressentimento, sendo necessária a inversão dos que compõe esta última) se relacionam com os tipos de orientação e suas subfunções, executou-se uma correlação momento-produto ( $r$ de Pearson). Os resultados encontram-se descritos na Tabela 1.

De acordo com a Tabela 1, é possível verificar que a dimensão benevolência e o fator geral apresentam correlações positivas e significativas, com quatro e duas subfunções valorativas, respectivamente. A dimensão benevolência com as subfunções interativa $(r=0,26 ; p<0,001)$; normativa $(r=0,19 ; p<0,001)$; suprapessoal $(r=0,16 ; p<0,001)$; e existência $(r=0,14 ; p=$ $0,01)$. E o fator geral com interativa $(r=0,23$; 
Tabela 1

Correlatos entre Valores Humanos e Perdão Conjugal

\begin{tabular}{lcccc}
\hline Valores Humanos & & Benevolência & Evitação-Ressentimento & $\begin{array}{c}\text { Fator Geral } \\
\text { (Perdão Conjugal) }\end{array}$ \\
\hline Social & $M(D P)$ & $4,46(1,40)$ & $3,34(1,39)$ & $4,55(1,18)$ \\
$\quad$ Interativa & $5,66(0,79)$ & $0,26^{* *}$ & 0,04 & $0,17^{*}$ \\
$\quad$ Normativa & $5,66(0,86)$ & $0,26^{* *}$ & $-0,13^{*}$ & $0,23^{* *}$ \\
Central & $5,64(1,02)$ & $0,19^{* *}$ & 0,03 & 0,09 \\
$\quad$ Suprapessoal & $5,68(0,79)$ & $0,17^{*}$ & 0,001 & 0,20 \\
Existência & $5,42(0,85)$ & $0,16^{* *}$ & $-0,05$ & $0,13^{*}$ \\
Pessoal & $5,94(0,92)$ & $0,14^{*}$ & 0,05 & 0,05 \\
Experimentação & $4,66(0,92)$ & 0,10 & 0,06 & 0,02 \\
Realização & $4,78(1,01)$ & 0,07 & 0,03 & 0,01 \\
\hline
\end{tabular}

Nota. $M=$ média; $D P=$ desvio padrão.

$* p<0,05 ; * * p<0,001$.

$p<0,001)$ e suprapessoal $(r=0,13 ; p=0,03)$.

Já a dimensão evitação - ressentimento, por sua vez, exibiu correlação expressiva somente com a subfunção interativa $(r=-0,13 ; p=0,02)$, sendo esta inversa.

Quanto aos tipos de orientação, constatou-se que a dimensão benevolência se correlacionou, positiva e significativamente, com dois tipos de orientação [social ( $r=0,26 ; p<0,001)$ e central $(r=0,17 ; p=0,01)]$; diferentemente da dimensão evitação - ressentimento que não apresentou nenhuma relação expressiva com os mesmos. Já o fator geral exibiu uma correlação, significativa e positiva, apenas com o tipo de orientação $s o$ $\operatorname{cial}(r=0,17 ; p=0,01)$.

\section{Valores Humanos como Preditores do Perdão Conjugal}

Com o intuito de verificar o poder preditivo dos tipos de orientação e das subfunções valorativas sobre o perdão conjugal, realizaramse duas análises de Regressão Linear Múltipla com método Stepwise (por etapas), por diminuir a incidência de problemas de multicolinearidade das variáveis (Field, 2005). Na primeira análise, as variáveis preditoras foram as subfunções valorativas e na segunda os tipos de orientação. Em ambas se tomou como variável critério o fator geral, Perdão Conjugal. Os resultados destas análises encontram-se detalhados na Tabela 2.

Tabela 2

Regressões Múltiplas entre Valores Humanos e Perdão Conjugal

\begin{tabular}{lcccc}
\hline \multicolumn{1}{c}{ Preditores } & $R^{2}$ Ajustado & $F$ & $\beta$ Padronizado & $t$ \\
\hline $\begin{array}{l}\text { Tipos de Orientação } \\
\text { Valores Sociais } \\
\text { Subfunções } \\
\text { Interativa }\end{array}$ & 0,03 & $F(1 ; 303)=9,55$ & 0,17 & 0,001 \\
& 0,05 & $F(1 ; 303)=15,74$ & 0,22 & 3,97 \\
\hline
\end{tabular}


De acordo com a primeira análise, constatou-se que a subfunção interativa $[F(1 ; 303)$ $=15,74 ; \mathrm{p}<0,001]$ explica $5 \%$ da variância total do perdão conjugal. Contribuindo de forma direta $(\beta$ Padronizado $=0,22)$ na explicação da concessão do perdão entre casais, exibindo indicadores estatisticamente significativos $(p<$ $0,05)$. A segunda regressão revelou que os valores sociais $[F(1 ; 303)=9,55 ; p<0,001]$ explicam $3 \%$ da remissão matrimonial, tratando-se de uma explicação estatisticamente significativa $(t>$ $1,96)$ e de ordem direta $(\beta$ Padronizado $=0,17)$.

\section{Perdão Conjugal e Variáveis Sociodemográficas}

Visando conhecer se há diferenças entre as médias das dimensões quanto às variáveis sexo, escolaridade, idade, tempo de relacionamento e grau de religiosidade foram executadas MANOVA's, apoiadas no teste de homogeneidade das matrizes de variância-covariância (M de $B O X)$, o qual teve um valor-p associado não significativo $(p=0,85)$. Logo, mesmo os grupos apresentando amostras com tamanhos diferentes, a suposição de homogeneidade foi garantida. Dessa forma, em relação à variável sexo, observou-se que esta influenciou as pontuações dos participantes nas duas dimensões [Lambda de Wilks $=0,94, F(2,306)=9,09 ; p<0,001$; $\left.\eta^{2}=0,06\right]$. Especificamente, as pessoas do sexo feminino pontuaram mais alto $(M=3,51 ; D P=$ $1,45)$ do que as do sexo masculino $(M=3,00$; $D P=1,24)$ na dimensão evitação - ressentimento $[F(1,307)=9,14 ; p=0,003]$; um padrão contrário foi observado para a dimensão benevolência em que os homens pontuaram mais alto $(M=4,91 ; D P=1,30)$ do que o fizeram as mulheres $(M=4,24 ; D P=1,40)[F(1,307)=$ $15,90 ; p<0,001]$.

Quanto à escolaridade [Lambda de Wilks = 0,$\left.91 ; F(12,602)=2,29 ; p=0,007 ; \eta^{2}=0,04\right]$, verificou-se diferença apenas na dimensão evitação-ressentimento $[F(6,302)=3,12 ; p=0,006]$. Especificamente, comparando os grupos (Teste Post Hoc de Bonferroni), constatou-se que as pessoas com Ensino Fundamental Incompleto $(M=4,38 ; D P=1,54)$ pontuaram mais alto do que as do Ensino Superior Completo $(M=3,16$; $D P=1,28)$ e Pós-Graduação $(M=3,04 ; D P=$ 1,38). Já em relação ao tempo de união [Lambda de Wilks $=0,97 ; F(10,544)=0,75 ; p=0,67 ; \eta^{2}$ $=0,01]$, idade [Lambda de Wilks $=0,99 ; F(4$, $\left.610)=0,71 ; p=0,59 ; \eta^{2}=0,005\right]$ e percepção quanto ao nível de religiosidade [Lambda de Wilks $=0,99 ; F(2,229)=0,45 ; p=0,64$; $\left.\eta^{2}=0,004\right]$, não foram observadas nenhuma influência destas variáveis nas médias dos participantes nas dimensões do perdão conjugal.

\section{Discussão}

Com base nos resultados, pode-se constatar que os valores sociais, como prenunciado nas análises de correlação, se apresentaram como bons preditores da remissão conjugal, corroborando a hipótese 1. Este achado vai ao encontro dos estudos acerca dos comportamentos pró-sociais (Dalbert, 2002; Strelan, 2007), uma vez que a subfunção interativa representa indivíduos que manifestam preocupação com o outro, exprimindo sentimentos de cuidado e afeto; e a subfunção normativa é característica de pessoas religiosas, que cumpre seus deveres, promovem a harmonia social (Gouveia, 2003, 2013) e fomentam relações mais próximas e bemajustadas, evitando sentimentos de evitação e ressentimento (Gordon \& Baucom, 1998). Nesse sentido, as pessoas guiadas por valores sociais encontram-se mais predispostas a conceder o perdão, corroborando com os achados de Strelan e McKee (2014).

Além disso, constatou-se que a subfunção que melhor explicou a remissão dentro dos relacionamentos conjugais foi a subfunção interativa, sustentando a hipótese anteriormente discutida. Este achado pode ser elucidado pela natureza desta subfunção, que é essencial para o estabelecimento, regulação e manutenção das relações interpessoais (Gouveia, 2013), a exemplo do casamento (Hook, Worthington, Utsey, Davis, \& Bumette, 2012).

Os valores centrais e suas subfunções não se revelaram bons preditores do perdão conjugal, refutando hipótese 2. Contudo, foi possível verificar que a subfunção existência apresentou 
uma correlação positiva e significativa com a dimensão benevolência e o fator geral (Perdão Conjugal). Segundo alguns autores (Lawler-Row, Scott, Rains, Edlis-Matityahou, \& Moore, 2007; Younger, Piferi, Jobe, \& Lawler, 2004), as vítimas de ofensas podem compreender o perdão como um fator que venha a lhe proporcionar um bem-estar maior quando comparado com situações de intensa vivência de sentimentos negativos frente ao agressor, chegando a compreender a remissão como uma autocura.

A subfunção suprapessoal, por sua vez, revelou uma correlação positiva com a dimensão benevolência. Esta relação pode ser justificada em decorrência da natureza teórica da própria subfunção que expressa a importância de ideias abstratas (Inglehart, 1991), enfatizando o pensar de forma mais geral e ampla (Schwartz, 1992). Desta forma, o perdão pode ser visto como processo intrapessoal que conduz os sujeitos à autorrealização (Braithwaite \& Law, 1985).

Os achados apontaram que os valores pessoais, bem como suas subfunções (experimentação e realização), não se caracterizaram como bons explicadores para a remissão matrimonial, refutando a hipótese 3 , além de não apresentarem relações expressivas com o perdão conjugal e suas dimensões. Não obstante, era esperado que as pessoas guiadas por valores pessoais apresentassem baixa habilidade de conceder o perdão aos seus parceiros, devido ao egocentrismo característico dos indivíduos que se pautam nesses valores (Gouveia, 2013; Gouveia, Fonsêca, Milfont, \& Fischer, 2011), conjeturando, assim, pouca disposição para perdoar, em decorrência de sua ínfima preocupação com os outros (Strelan \& McKee, 2014).

Acredita-se que os achados não foram ao encontro da literatura apontada em decorrência da característica amostral dos sujeitos da presente pesquisa, os quais relataram, em sua maioria, possuir um tempo de união variando entre 1 e 3 anos de casamentos. Pois, segundo Wagner, Ribeiro, Arteche e Bornholdt (1999), o início do matrimônio é marcado por esforços de ambos os parceiros para fortalecer a união e o amor, chegando muitas vezes a deixar de lado os seus desejos pessoais para pensar no bem estar do casal, refletindo o aumento da tolerância, compreensão fácil e perdão súbito.

Por fim, observar-se que os valores supramencionados e discutidos das relações são considerados estatisticamente fracos (Menor que 0,30 ), a partir das magnitudes consideradas (Cohen, 1998). Não obstante, leva-se em conta a natureza maleável dos construtos abordados e que segundo Hemphill (2003), encontrar correlações acima de 0,30 na psicologia parece ser uma exceção do que uma regra. Merecendo os resultados, assim, serem encarados como exploratórios e passiveis de replicações.

Quanto às variáveis sociodemográficas (sexo, escolaridade, idade, tempo de união, ao nível de religiosidade), verificou-se que apenas o sexo e a escolaridade influenciaram significativamente às médias dos sujeitos nas dimensões do perdão conjugal, corroborando apenas as hipóteses 4 e 8 . Em relação ao sexo constatou-se que os homens são mais benevolentes e as mulheres mais predispostos a emitir comportamentos de evitação e ressentimento.

Esse padrão também foi encontrado no estudo de Sales (2014), porém o mesmo não condiz com a maioria dos achados literários (Mullet, Houdbine, Laumonier, \& Girard, 1998; McCullough, Bellah, Kilpatrick, \& Johnson, 2001), que apontam que as mulheres são mais predispostas a conceder a remissão, tendo em vista o seu desejo de estabelecer e manter relações harmoniosas dentro de suas interações. Já os homens, apresentam um perfil mais vingativo, sendo capazes de exprimir comportamentos agressivos e fazer justiça com suas próprias mãos. Entretanto, é necessário levar em consideração o contexto cultural, nordeste brasileiro, no qual as pesquisas foram realizadas; pois as diferenças comportamentais frente ao perdão, provavelmente, variam de cultura para cultura (Sandage \& Williamson, 2005).

Em relação à escolaridade, foi encontrada diferença apenas na dimensão negativa, cujos sujeitos com ensino fundamental incompleto pontuaram mais alto do que as do Ensino Superior Completo e Pós-Graduação. Expressando assim, a influência da maturidade adquirida ao longo da formação acadêmica (Souza \& Wechs- 
ler, 2013), sendo este um fator de grande importância na decisão de perdoar ou não (Allemand $\&$ Steiner, 2012).

Quanto à variável idade, destaca-se que o resultado corroborou com o estudo de Sandage, Worthington, Hight, e Berry (2000), em que não foi encontrada nenhuma relação entre idade e perdão. Não obstante, alguns autores (Allemand, 2008; Ghaemmaghami, Allemand, \& Martin, 2011; Steiner, Allemand, \& McCullough, 2012) pontuam que pessoas mais velhas tendem a apresentar uma maior habilidade de conceder o perdão do que aquelas mais jovens, contrapondo-se a hipótese 5. Isso aconteceria em decorrência das vivências, em maior número, de problemas interpessoais e transgressões ao longo da vida (Allemand \& Steiner, 2012), levando as pessoas com mais experiência a direcionarem sua atenção a aspectos emocionais mais significativos, como a manutenção de relacionamentos, concedendo assim, com maior facilidade o perdão ao seu ofensor (Lang \& Carstensen, 2002).

O fator religiosidade também não apresentou influência nas médias dos participantes nas dimensões do perdão conjugal, refutando a hipótese 6. Esse resultado se assemelhou ao estudo de Rique e Camino (2010), os quais constataram uma ausência de relacionamento entre diferentes níveis de religiosidade e o perdão em uma amostra brasileira. Tal fato pode ter ocorrido em decorrência da utilização de apenas um único item (Gouveia et al., 2009), semelhante ao empregado na presente pesquisa. Entretanto, era esperado que as pessoas com maior nível de religiosidade seriam mais propensas a concederem o perdão, tendo em vista sua crença no casamento como algo sagrado e sua dissolução como um ato de desrespeito a ordem divina (Krumrei, Mahoney, \& Pargament, 2011).

Além disso, destaca-se que o tempo de união também não revelou nenhuma diferença significativa entre as médias das dimensões benevolência e evitação-ressentimento, divergindo de alguns estudos (Guilford \& Bengton, 1979; Rollins \& Feldman, 1970), os quais apontam que aqueles que possuíssem um menor (1- 3 anos) e um maior tempo de união (acima de 17 anos;
Guilford \& Bengton, 1979; Rollins \& Feldman, 1970) fossem mais predispostos a perdoar o parceiro ao decorrer do tempo e em diferentes situações, visando à harmonia e a manutenção do relacionamento de longa data, não corroborando a hipótese 7.

Frente aos resultados, acredita-se que os valores sociais se constituem importantes preditores para o perdão conjugal. Fornecendo assim, arcabouços empíricos para o desenvolvimento de técnicas e intervenções clínicas, que visem solucionar problemas e/ou conflitos provenientes das interações conjugais. Mesmo alcançando os objetivos propostos, não se pode deixar de apontar possíveis limitações. Por exemplo, utilizou-se uma amostra por conveniência (não probabilística; Cozby, 2003), que implica na restrição de generalização dos achados. Todavia, isso não invalida ou desmerece os resultados, uma vez que o estudo não tinha tal desígnio.

Outro ponto importante a ponderar é a possibilidade de as respostas terem sido influenciadas pela desejabilidade social, isto é, as pessoas podem ter se mostrado mais dispostas a perdoar do que realmente são, emitindo assim, respostas mais favorável ou aceitável socialmente. Sendo este padrão comum em medidas de autorrelato (Anastasi \& Urbina, 2000), utilizado no presente estudo (Escala de Perdão Conjugal e Questionário de Valores Básicos).

Diante dos resultados apresentados, confia-se que o presente artigo contribui para a literatura dos construtos aqui abordados, contudo dadas às limitações apresentadas neste trabalho, sugere-se que novas pesquisas acerca desta temática sejam desenvolvidas. Quanto aos estudos futuros, aconselha-se a utilização de amostras maiores e mais diversificadas, selecionada de forma randômica, reunindo indivíduos de vários estados brasileiros, pois se entende que a orientação cultural das pessoas pode variar de uma a outra região no Brasil (Gouveia, Albuquerque, Clemente, \& Espinosa, 2002; Hofstede, Hofstede, \& Minkov, 2010), podendo ter impacto no perdão, sobretudo no âmbito das relações conjugais, e nos valores humanos. 


\section{Referências}

Abreu, E. L. (2013). A relação entre o pensamento moral da justiça e o pensamento moral do perdão (Dissertação de mestrado, Departamento de Psicologia, Universidade Federal da Paraíba, João Pessoa, PB, Brasil). Recuperado em http:// tede.biblioteca.ufpb.br/bitstream/tede/6917/1/ arquivototal.pdf

Allemand, M. (2008). Age differences in forgivingness: The role of future time perspective. Journal of Research in Personality, 42(5), $1137-$ 1147. doi:10.1016/j.jrp.2008.02.009

Allemand, M., \& Steiner, M. (2012). Situation-specific forgiveness and dispositional forgiveness: A lifespan development perspective. In E. Kahls \& J. Maes (Eds.), Justice psychology and conflicts: Theoretical and empirical contributions (pp. 361-375). New York: Springer.

Allemand, M., Steiner, M., \& Hill, P. L. (2013). Effects of a forgiveness intervention for older adults. Journal of Counseling Psychology, 60(2), 279-286. doi:10.1037/a0031839

Anastasi, A., \& Urbina, S. (2000). Testagem psicológica. Porto Alegre, RS: Artes Médicas.

Barbosa, L. H. G. M. (2015). Explicando a disposição para perdoar: O papel dos valores humanos e das crenças ao mundo justo/injusto (Dissertação de mestrado, Departamento de Psicologia, Universidade Federal da Paraíba, João Pessoa, PB, Brasil). Recuperado em http:// bdtd.biblioteca.ufpb.br/bitstream/tede/7565/2/ arquivototal.pdf

Bardi, A., \& Schwartz, S. H. (2003). Values and behavior: Strength and structure of relations. Personality and Social Psychology Bulletin, 29(10), 1207-1220. doi:10.1177/0146167203254602

Braithwaite, V. A., \& Law, H. G. (1985). Structure of human values: Testing the adequacy of the Rokeach Value Survey. Journal of personality and social psychology, 49(1), 250-263. doi:10.1037/0022-3514.49.1.250

Braithwaite, S. R., Selby, E. A., \& Fincham, F. D. (2011). Forgiveness and relationship satisfaction: Mediating mechanisms. Journal of $\mathrm{Fa}$ mily Psychology, 25(4), 551-559. doi:10.1037/ a0024526

Brock, R. L., Barry, R. A., Lawrence, E., Dey, J., \& Rolffs, J. (2012). Internet administration of paper-and-pencil questionnaires used in couple research: Assessing psychometric equivalence. Assessment, 19(2), 226-242. doi:10.1177/1073191110382850

Bueno, R. K., Souza, S. A., Monteiro, M. A., \& Teixeira, R. H. M. (2013). Processo de diferenciação dos casais de suas famílias de origem. Psico, 44(1), 16-25. Recuperado em http://revistaseletronicas.pucrs.br/ojs/index.php/revistapsico/ article/viewArticle/9420

Calo-Blanco, A. (2014). Fairness, freedom, and forgiveness in health care. Social Choice and Welfare, 43(1), 141-151. doi:10.1007/s00355013-0774-7

Chung, M. S. (2014). Pathways between attachment and marital satisfaction: The mediating roles of rumination, empathy, and forgiveness. Personality and Individual Differences, 70(6), 246-251. doi:10.1016/j.paid.2014.06.032

Cohen, J. (1988). Statistical power analysis for the behavioral sciences $\left(2^{\text {nd }}\right.$ ed.). Hillsdale, NJ: Erlbaum.

Cozby, P. C. (2003). Métodos de pesquisa em ciências do comportamento. São Paulo, SP: Atlas.

Dalbert, C. (2002). Beliefs in a just world as a buffer against anger. Social Justice Research, 15(2), 123-145. doi:10.1023/A:1019919822628.

Downie, R. S. (1965). Forgiveness. Philosophical Quarterly, 15(59), 128-134. doi:10.2307/ 2218212

Enright, R. D., \& Coyle, C. T. (1998). Researching the process model of forgiveness within psychological interventions. In E. L. Worthington (Ed.), Dimensions of forgiveness: Psychological research and theological perspectives (pp. 139161). Philadelphia, PA: Templeton Press.

Enright, R. D., Rique, J., \& Coyle, C. T. (2000). Enright forgiveness inventory user's manual, Redwood City, CA: Mind Garden.

Field, A. (2005). Discovering Statistics Using SPSS $\left(2^{\text {nd }}\right.$ ed.). London: Sage.

Fife, S. T., Weeks, G. R., \& Stellberg-Filbert, J. (2013). Facilitating forgiveness in the treatment of infidelity: An interpersonal model. Journal of Family Therapy, 35(4), 343-367. doi:10.1111/ j.1467-6427.2011.00561.x

Fincham, F. D., \& Beach, S. R. H. (2002). Forgiveness in marriage: Implications for psychological aggression and constructive communication. Personal Relationships, 9(3), 239-251. doi:10.1111/1475-6811.00016 
Fincham, F. D., Beach, S. R., \& Davila, J. (2004). Forgiveness and conflict resolution in marriage. Journal of Family Psychology, 18(1), 72-81. doi:10.1037/0893-3200.18.1.72

Fincham, F. D., Beach, S. R., \& Davila, J. (2007). Longitudinal relations between forgiveness and conflict resolution in marriage. Journal of Family Psychology, 21(3), 542-545. doi:10.1037/08933200.21.3.542

Fincham, F. D., Hall, J., \& Beach, S. R. (2006). Forgiveness in marriage: Current status and future directions. Family Relations, 55(4), 415-427. doi:10.1111/j.1741-3729.2005.callf.x-i1

Fincham, F. D., Paleari, F. G., \& Regalia, C. (2002). Forgiveness in marriage: The roles of relationship quality, attributions and emotions. Personal Relationships, 9(1), 27-37.

Freire, L. A. (2015). Atitudes frente á homoparentalidade: Uma explicação a partir de variáveis explícitas e implícitas (Tese de doutorado, Departamento de Psicologia, Universidade Federal da Paraíba, João Pessoa, PB, Brasil). Recuperado em http://tede.biblioteca.ufpb.br/bitstream/ tede/7521/2/arquivototal.pdf

Ghaemmaghami, P., Allemand, M., \& Martin, M. (2011). Forgiveness in younger, middle-aged and older adults: Age and gender matters. Journal of Adult Development, 18(4), 192-203. doi:10.1007/s10804-011-9127-x

Gordon, K. C., \& Baucom, D. H. (1998). Understanding betrayals in marriage: A synthesized model of forgiveness. Family Process, 37(4), 425-449. doi:10.1111/j.1545-5300.1998.00425.x

Gouveia, V. V. (1998). La naturaleza de los valores descriptores del individualismo e del colectivismo: una comparación intra e intercultural (Doctoral dissertation, Department of Psychology, Complutense University of Madri, Spain).

Gouveia, V. V. (2003). A natureza motivacional dos valores humanos: Evidências acerca de uma nova tipologia. Estudos de Psicologia (Natal), 8(3), 431-443. doi:10.1590/S1413$-294 X 2003000300010$

Gouveia, V. V. (2013). Teoria Funcionalista dos Valores Humanos: Fundamentos, aplicações e perspectivas. São Paulo, SP: Casa do Psicólogo.

Gouveia, V. V., Albuquerque, F. J. B., Clemente, M., \& Espinosa, P. (2002). Human values and social identities: A study in two collectivist cultures. International Journal of Psychology, 37(6), 333342. doi:10.1080/00207590244000179
Gouveia, V. V., Fonseca, P. N., Milfont, T. L., \& Fischer, R. (2011). Valores humanos: Contribuições e perspectivas teóricas. In C. V. Torres \& E. R. Neiva (Eds.), A psicologia social: Principais temas e vertentes (pp. 296-313). Porto Alegre, RS: ArtMed.

Gouveia, V. V., Gonçalves, M. P., Costa, J. D. M., Araújo, L. B. U. D., Gouveia, R. S., \& Medeiros, E. D. D. (2009). Disposição para perdoar, desejabilidade social e religião: Um estudo correlacional. Revista Bioética, 17(2)-297-308.

Gouveia, V.V., Milfont, T. L., \& Guerra, V. M. (2014). Functional theory of human values: Testing its content and structure hypotheses. Personality and Individual Differences, 60, 41-47. doi:10.1016/j.paid.2013.12.012

Guilford, R., \& Bengtson, V. (1979). Measuring marital satisfaction in three generations: Positive and negative dimensions. Journal of Marriage and the Family, 41(2), 387-398. doi:10.2307/351705

Hall, J. H., \& Fincham, F. D. (2006). Relationship dissolution following infidelity: The roles of attributions and forgiveness. Journal of Social and Clinical Psychology, 25(5), 508-522. doi:10.1521/jscp.2006.25.5.508

Hemphill, J. F. (2003). Interpreting the magnitudes of correlation coefficients. American Psychologists, 58(1), 78-80. Retrieved from https://www. ncbi.nlm.nih.gov/pubmed/12674822

Hofstede, G., Hofstede, G. J., \& Minkov, M. (2010). Cultures and organizations: Software of the mind. London: McGraw-Hill.

Hook, J. N., Worthington, E. L., Utsey, S. O., Davis, D. E., \& Burnette, J. L. (2012). Collectivistic self-construal and forgiveness. Counseling and Values, 57(1), 109-124. doi:10.1002/j.2161007X.2012.00012.X

Inglehart, R. (1977). The silent revolution: Changing values and political styles among Western publics. Princeton, NJ: Princeton University Press.

Inglehart, R. (1991). El cambio cultural en las sociedades industriales avanzadas. Madri: Siglo XXI.

Krumrei, E. J., Mahoney, A., \& Pargament, K. I. (2011). Spiritual stress and coping model of divorce: A longitudinal study. Journal of $\mathrm{Fa}$ mily Psychology, 25(6), 973-985. doi:10.1037/ a0025879

Lander, I. (2012). Towards the incorporation of forgiveness therapy in healing the complex trauma 
of parental incarceration. Child and Adolescent Social Work Journal, 29(1), 1-19. doi:10.1007/ s10560-011-0248-7

Lang, F. R., \& Carstensen, L. L. (2002). Time counts: Future time perspective, goals, and social relationships. Psychology and aging, 17(1), 125139. doi:10.1037/0882-7974.17.1.125

Lawler-Row, K. A., Scott, C. A., Raines, R. L., Edlis-Matityahou, M., \& Moore, E. W. (2007). The varieties of forgiveness experience: Working toward a comprehensive definition of forgiveness. Journal of Religion and Health, 46(2), 233-248. doi:10.1007/s10943-006-9077-y

Lin, Y., Worthington, E. L., Griffin, B. J., Greer, C. L., Opare-Henaku, A., Lavelock, C. R., ...Muller, H. (2014). Efficacy of reach forgiveness across cultures. Journal of Clinical Psychology, 70(9), 781-793. doi:10.1002/jclp.22073

Lopes, B. J., Fonsêca, P. N., Medeiros, E. D., Almeida, A. C., \& Gouveia, V. V. (2016). Escala de perdão conjugal (MOFS): Evidências de validade de construto no contexto brasileiro Psico, 47(2), 121-131. doi:10.15448/19808623.2016.2.22439

Maslow, A. H. (1954). Motivation and personality. New York: Harper and Row.

McCullough, M. E., Bellah, G. C., Kilpatrick, S. D., \& Johnson, J. L. (2001). Vengefulness: Relationships with forgiveness, rumination, well-being, and the Big Five. Personality and Social Psychology Bulletin, 27(5), 601-610. doi:10.1177/0146167201275008

McCullough, M. E., Rachal, K., Sandage, S., Worthington, E. L., Brown, S., \& Hight, T. (1998). Interpersonal forgiving in close relationships: II. Theoretical elaboration and measurement. Journal of Personality and Social Psychology, 75(6), 1586-1603. doi:10.1037/0022-3514.75.6.1586

Medeiros, E. D. (2011). Teoria Funcionalista dos Valores Humanos: Testando sua adequação intra e interculturalmente (Doctoral dissertation, Departamento de Psicologia, Universidade Federal da Paraíba, João Pessoa, PB, Brazil).

Melo, R. L. P. (2014). Correlatos valorativos das atitudes frente à aposentadoria (Dissertação de mestrado, Departamento de Psicologia, Universidade Federal da Paraíba, João Pessoa, PB, Brasil). Recuperado em http://www.vvgouveia. net/index.php? option=com_phocadownload\&v iew $=$ category $\& \mathrm{id}=18 \&$ Itemid $=175 \&$ lang $=$ pt-br
Monteiro, R. P. (2014). Entendendo a Psicopatia: Contribuição dos traços de personalidade $e$ valores humanos (Dissertação de mestrado, Departamento de Psicologia, Universidade Federal da Paraíba, João Pessoa, PB, Brasil). Recuperado em http://tede.biblioteca.ufpb.br/bitstream/ tede/7564/2/arquivototal.pdf

Mullet, E., Houdbine, A., Laumonier, S., \& Girard, M. (1998). "Forgiveness:" Factor structure in a sample of young, middle-aged, and elderly adults. European Psychologist, 3(4), 289-297. doi:10.1002/9781118339893.wbeccp228

Nascimento, B. S. (2015). Atitudes frente à violência contra a mulher: $O$ papel dos valores e da desumanização da mulher (Dissertação de mestrado, Departamento de Psicologia, Universidade Federal da Paraíba, João Pessoa, PB, Brasil). Recuperado em http://tede.biblioteca.ufpb.br/ bitstream/tede/7643/2/arquivototal.pdf

North, J. (1998). The "ideal" of forgiveness: A philosopher's exploration. In R. D. Enright \& J. North (Eds.), Exploring forgiveness (pp. 15-45). Madison, WI: University of Wisconsin Press.

Paleari, F. G., Regalia, C., \& Fincham, F. D. (2009). Measuring offence-specific forgiveness in marriage: The Marital Offence-Specific Forgiveness Scale (MOFS). Psychological Assessment, 21(2), 194-209. doi:10.1037/a0016068

Peets, K., Hodges, E. V., \& Salmivalli, C. (2013). Forgiveness and its determinants depending on the interpersonal context of hurt. Journal of Experimental Child Psychology, 114(1), 131-145. doi:10.1016/j.jecp.2012.05.009

Pelucchi, S., Paleari, F. G., Regalia, C., \& Fincham, F. D. (2013). Self-forgiveness in romantic relationships: It matters to both of us. Journal of $\mathrm{Fa}$ mily Psychology, 27(4), 541-549. doi:10.1037/ a0032897

Rique, J., \& Camino, C. P. S. (2010). O perdão interpessoal em relação a variáveis psicossociais e demográficas. Psicologia Reflexão e Crítica, 23(3), 525-532.

Rokeach, M. (1973). The nature of human values. New York: Free Press.

Rollins, B. C., \& Feldman, H. (1970). Marital satisfaction over the family life cycle. Journal of Marriage and the Family, 32(1), 20-28. doi:10.2307/349967

Sales, H. F. S. (2014). Disposição para perdoar e sua associação com saúde geral e variáveis 
sociodemográficas (Trabalho de conclusão de curso, Departamento de Psicologia, Universidade Federal do Píaui, Parnaiba, PI, Brasil).

Sandage, S. J., \& Williamson, I. (2005). Forgiveness in cultural context. In E. L. Worthington (Ed.), Handbook of forgiveness (pp. 41-55). New York: Brunner-Routledge.

Sandage, S. J., Worthingon, E. L., Jr., Hight, T. L., \& Berry, J. W. (2000). Seeking forgiveness: Theoretical context and an initial empirical study. Journal of Psychology and Theology, 28(1), 21-35. doi:10.091-6471/410-730

Schwartz, S. H. (1992). Universal in the content and structure of values: Theoretical advances and empirical tests in 20 countries. In M. P. Zanna (Ed.), Advanced in experimental social psycho$\log y$ (pp. 1-65). New York: Academic Press.

Souza, A. A. F., \& Wechsler, S. M. (2013). Inteligência e criatividade na maturidade e velhice. Psicologia: Reflexão e Crítica, 26(4), 643-653. Recuperado em http://www.scielo.br/pdf/prc/ v26n4/04.pdf

Steiner, M., Allemand, M., \& McCullough, M. E. (2012). Do agreeableness and neuroticism explain age differences in the tendency to forgive others? Personality and Social Psychology Bulletin, 38(4), 441-453. doi:10.1177/0146167211427923

Strelan, P. (2007). The prosocial, adaptive qualities of just world beliefs: Implications for the relationship between justice and forgiveness. Personality and Individual Differences, 43(4), 881-890. doi:10.1016/j.paid.2007.02.015

Strelan, P., \& McKee, I. (2014). Inclusive justice beliefs and forgiveness: Commonality through self-transcending values. Personality and Individual Differences, 68, 87-92. doi:10.1016/j. paid.2014.04.001

Strelan, P., McKee, I., Calic, D., Cook, L., \& Shaw, L. (2013). For whom do we forgive? A functional analysis of forgiveness. Personal Relationships, 20(1), 124-139. doi:10.1111/j.14756811.2012.01400.x
Toussaint, L. L., Owen, A. D., \& Cheadle, A. (2012). Forgive to live: Forgiveness, health, and longevity. Journal of Behavioral Medicine, 35(4), 375-386. doi:10.1007/s10865-011-9362-4

Wagner, A., \& Mosmann, C. (2012). Intervenção na conjugalidade: Estratégias de resolução de conflitos conjugais. In. M. N. Baptista \& M. L. M. Teodoro (Eds.), Psicologia de família: Teoria, avaliação e intervenções (pp. 240-248). Porto Alegre, RS: Artmed.

Wagner, A., Ribeiro, L. D. S., Arteche, A. X., \& Bornholdt, E. A. (1999). Configuração familiar e o bem-estar psicológico dos adolescentes. Psicologia: Reflexão e Crítica, 12(1), 147-156. doi:10.1590/S0102-79721999000100010

Witvliet, V. O. C. (2001). Forgiveness and health: Review and reflections on a matter of faith, feelings, and physiology. Journal of Psychology and Theology, 29(3), 212. Recuperado em http:// go.galegroup.com/ps/i.do? id=GALE\%7CA797 $57933 \& \mathrm{v}=2.1 \& \mathrm{u}=$ capes $\& \mathrm{it}=\mathrm{r} \& \mathrm{p}=\mathrm{AONE} \& \mathrm{sw}=$ w\&asid $=3$ ba970013b90e1dea857b9505a375a2f

Worthington, E. L. (2003). Forgiving and reconciling: Bridges to wholeness and hope. Downers Grove, IL: Inter Varsity Press.

Worthington, E. L. (2005). Handbook of forgiveness. New York: Routledge.

Younger, J. W., Piferi, R. L., Jobe, R. L., \& Lawler, K. A. (2004). Dimensions of forgiveness: The views of laypersons. Journal of Social and Personal Relationships, 21(6), 837-855. doi: $10.1177 / 0265407504047843$ 